\title{
Future of Orthodontics: Let Us Look Before We Leap- A Review Article
}

\author{
Shathriya $\mathrm{K}^{1 *}$, Bejoy P Unni ${ }^{2}$, Lakshmi Lakshmanan ${ }^{3}$, Vinith Joje ${ }^{4}$, Sanju Thomas ${ }^{5}$ \\ ${ }^{1}$ Post Graduate Student, Department of Orthodontics and Dentofacial Orthopedics, Malabar Dental College, Kerala, India \\ ${ }^{2}$ Professor \& HOD, Department of Orthodontics and Dentofacial Orthopedics, Malabar Dental College, Kerala, India \\ ${ }^{3,4}$ Reader, Department of Orthodontics and Dentofacial Orthopedics, Malabar Dental College, Kerala, India \\ ${ }^{5}$ Senior Lecturer, Department of Orthodontics and Dentofacial Orthopedics, Malabar Dental College, Kerala, India
}

DOI: $10.36348 /$ SJODR.2019.v04i10.006

| Received: 11.10.2019| Accepted: 18.10.2019| Published: 21.10.2019

*Corresponding author: Dr. Shathriya K

\section{Abstract}

The demand of aesthetic orthodontics has increased among young and adult population. The request for fast and invisible orthodontic treatment has encouraged the development of clear aligners. Clear aligners are considered as an alternative to dental braces. They are devices that use thermoplastic materials which apply pressure to teeth to move into the desired position. Dozens of company around world offer some type of clear aligner orthodontic product and is widely considered as the future of Orthodontics. Advantages of sequential aligner therapy has been discussed widely, this article discuss concerns and adverse effects and reactions associated with aligner therapy. However, until more clinical research becomes available, aligners cannot be routinely prescribed as an effective alternative to fixed labial appliances. Let us look before we leap.

Keywords: Bpa-bisphenol-a, invisalign.

Copyright @ 2019: This is an open-access article distributed under the terms of the Creative Commons Attribution license which permits unrestricted use, distribution, and reproduction in any medium for non-commercial use (NonCommercial, or CC-BY-NC) provided the original author and source are credited.

\section{INTRODUCTION}

In recent years, increasing number of adult patients have sought orthodontic treatment and expressed a desire for aesthetic and comfortable alternatives like lingual orthodontics and invisalign appliances to conventional fixed appliances. With the growing popularity of the aligners it is expected that more patients would be inclined to use it rather than traditional braces for correcting their malocclusions [1]. Prior to 1998 , orthodontic clear aligner treatment was predominantly for very minor tooth movement, usually at the end of orthodontic treatment or to treat minor alignment relapse. In 1998, Align Technology, Inc. introduced Invisalign to the orthodontic market. It used computerized 3D technology to visualize and move the teeth in a virtual model. This technology along with advances in $3 \mathrm{D}$ printing and manufacturing efficiencies allowed aligners to be produced in a large numbers and in a timely fashion. Initial cases were mild crowding or spacing [2] which progressed to cases that needed expansion and/or classification correction [3].

Each aligner moves teeth incrementally and is worn for about weeks, then replaced by the next one in series until the final position is achieved [4]. The aligners are removable and are made of $0.75 \mathrm{~mm}$-thick polyurethane. Each aligner is programmed to produce a precise movement on a tooth of about $0.15-0.25 \mathrm{~mm}$ [5]. Invisalign applies intermittent forces to the teeth just as most of the active removable appliances [4]. Orthodontic treatment with Invisalign aligners could lead to root resorption as any other orthodontic treatment [6]. A review by Rossini demonstrated that Invisalign is effective for simple malocclusion treatment [6].

Some of the advantages of the clear aligners include

- Approximating the treatment duration a little more precisely than braces.

- Avoiding extractions of premolars by creating interdental space via interproximal reduction.

- Less frequent trips to the dental chair by allowing patients to replace their aligners on their own every few weeks.

- Healthier periodontal tissue and less risk of enamel decalcification by avoiding brackets.

- Patients can easily remove the aligners when they eat and also during their usual hygiene routine of brushing and flossing.

- They are virtually invisible which is a big aesthetic benefit. 
The clear aligner technique is continually evolving because of research and development in materials, manufacturing techniques, auxiliaries, and computer programming of tooth movement. The aligners available today are vastly different than those available in 2000. Different types of aligners are available today throughout the world. However the biocompatibility of these materials with human soft tissues is still under research. In this article we will discuss the disadvantages of aligners and the problems that may arise during treatments with them.

\section{Clear aligners: are they really impeccable}

Clear aligners were first used only on simple orthodontic cases, but now they are used for even the most advanced orthodontic cases. Not only do we find that clear aligners take away the social stigma of orthodontics, but they also tend to be more efficient when it comes to patients oral health. OR ARE THEY??

\section{Disadvantages}

The clear aligners are limited in what they can accomplish. They are best suited to treat maloclussion that are considered mild to moderate [7]. They are not effective in controlling anterior buccolingual inclination, extrusion and rotation of teeth [8].

Patients with short crowns [9] or porcelain veneers may be limited from having invisible braces which require buttons or attachments since it can be problematic to bond these formations on to some type of dental work.

Since the plastic aligners should fit tightly to the shape of the teeth to allow enough force to shift them, the shape of the teeth may limit what the braces can accomplish. Teeth that are short, pegged, round or severely tipped may not be properly manipulated by such aligners.

All teeth restorations should be completed prior to treatments with invisible braces since even one dental treatment to alter the shape of the tooth can interfere with the fitting of the aligners.

Since the aligners fit snugly against the teeth, it is possible for saliva and bacteria to become trapped in the aligner. Since fresh saliva doesn't come in contact with teeth to wash away bacteria and debris when aligners are on, there is a risk of developing cavities. This may also lead to bad breathe and dry mouth.

Smoking, drinking red wine or coffee will lead to staining of aligners and adhesives which is a major disadvantage to its aesthetic nature [10].

Strict requirement of patient compliance is needed for successful outcome of treatment [11].

\section{HEALTH HAZARDS}

Effect on Oral epithelial cell: Premaraj conducted an invitro study to evaluate the cellular responses of oral epithelium exposed to invisalign plastic. The study indicated that gingival epithelial cell exposure to Invisalign plastic in a saline-solution based environment resulted in alteration of membrane integrity, lowered metabolic activity, and reduced cellto-cell contact by relaxed tight junctions; this leads to increased permeability of fluid flow [12].

\section{Release of BPA}

Plastic materials and auxiliaries such as adhesives and polycarbonate brackets and aligners release bisphenol A. It is an endocrine disruptor which may lead to obesity, fertility problems, heart diseases, increased diabetic risks and breast and prostate cancer [13].

\section{Presence of Dangerous Chemicals}

Invisalign, a type of clear aligner does not have the chemical composition to release bisphenol A. Invisalign plastic is a polyurethane plastic namely isocyanate which might pose certain health issues. Isocyanate usually causes mucous membrane irritation, asthmatic or hyper sensitivity reaction. Epithelial cell death, damage to the epithelial layer or loss of integrity could lead to hexamethylene diisocyanate conjugated protein exposure to the human immune system. As a result, it could create immunologic reactions [12].

\section{Analysis of the MAUDE Database}

Veerasathpurush conducted an analysis of the Manufacturer and User facility Device Experience (MAUDE) database of the United States Food and Drug Administration on the adverse clinical events reported during Invisalign treatment. The analysis revealed that patients reported adverse events like difficulty in breathing, followed by sore throat, swollen throat, swollen tongue, hives and itchiness, anaphylaxis, swollen lips, feeling of throat closing/ tight airway/ airway obstruction/ laryngospasm, chest pain cough, nausea, difficulty swallowing, dry mouth, head ache, swelling of eyes, blisters or sores of lips, fatigue, burning/tingling/sore tongue, blisters or ulcerations on tongue, swelling of gums, lymph node enlargement, rashes on face or body, sinus tachycardia, palpitations, numbness of tongue and chest congestion. All the patients believed that they experienced their reported symptoms after use of invisalign trays and the symptoms minimized or disappeared after discontinuation of aligners [1].

\section{CONCLUSION}

Orthodontic treatment, in some form is now provided by not only specialist orthodontists but also general dental practitioners. The above review indicates that further study of aligners is warranted, particularly 
in view of continuous exposure by the patient to the material during longer treatment protocols.

\section{REFERENCE}

1. Allareddy, V., Nalliah, R., Lee, M. K., Rampa, S., \& Allareddy, V. (2017). Adverse clinical events reported during Invisalign treatment: Analysis of the MAUDE database. American Journal of Orthodontics and Dentofacial Orthopedics, 152(5), 706-710.

2. Boyd, R. L., Miller, R. J., \& Vlaskalic, V. (2000). The Invisalign system in adult orthodontics: mild crowding and space closure cases. Journal of Clinical Orthodontics, 34(4), 203-212.

3. Boyd, R. L., \& Waskalic, V. (2001, December). Three-dimensional diagnosis andorthodontic treatment of complex malocclusions with the invisalign appliance. In Seminars in Orthodontics (Vol. 7, No. 4, pp. 274-293). WB Saunders.

4. Brezniak, N., \& Wasserstein, A. (2008). Root resorption following treatment with aligners. The Angle Orthodontist, 78(6), 1119-1124.

5. Houle, J. P., Piedade, L., Todescan Jr, R., \& Pinheiro, F. H. L. (2016). The predictability of transverse changes with Invisalign. The Angle Orthodontist, 87(1), 19-24.

6. Gay, G., Ravera, S., Castroflorio, T., Garino, F., Rossini, G., Parrini, S., ... \& Deregibus, A. (2017). Root resorption during orthodontic treatment with Invisalign $®$ : a radiometric study. Progress in orthodontics, 18(1), 12.

7. Joffe, L. (2003). Invisalign $®$ : early experiences. Journal of orthodontics, 30(4), 348352.
8. Kamatovic, M. (2004). A retrospective evaluation of the effectiveness of the Invisalign appliance using the PAR and irregularity indices [dissertation]. Toronto (Ont.): University of Toronto.

9. Phan, X., \& Ling, P. H. (2007). Clinical limitations of Invisalign. Journal of the Canadian Dental Association, 73(3).

10. Liu, C. L., Sun, W. T., Liao, W., Lu, W. X., Li, Q. W., Jeong, Y., ... \& Zhao, Z. H. (2016). Colour stabilities of three types of orthodontic clear aligners exposed to staining agents. International journal of oral science, 8(4), 246.

11. Bräscher, A. K., Zuran, D., Feldmann Jr, R. E., \& Benrath, J. (2017). Erratum to: Patient survey on Invisalign ${ }^{\circledR}$ treatment comparing the SmartTrack $®$ material to the previously used aligner material. Journal of orofacial orthopedics= Fortschritte der Kieferorthopadie: Organ/official journal Deutsche Gesellschaft fur Kieferorthopadie, 78(6), 511.

12. Premaraj, T., Simet, S., Beatty, M., \& Premaraj, S. (2014). Oral epithelial cell reaction after exposure to Invisalign plastic material. American Journal of Orthodontics and Dentofacial Orthopedics, 145(1), 64-71.

13. Kloukos, D., Pandis, N., \& Eliades, T. (2013). Bisphenol-A and residual monomer leaching from orthodontic adhesive resins and polycarbonate brackets: a systematic review. American journal of orthodontics and dentofacial orthopedics, 143(4), S104-S112. 